\title{
Characteristic temperature for the immiscible-miscible transition of binary condensates in optical lattices
}

\author{
K. Suthar ${ }^{1,2}$ and D. Angom ${ }^{1}$ \\ ${ }^{1}$ Physical Research Laboratory, Navrangpura, Ahmedabad-380009, Gujarat, India \\ ${ }^{2}$ Indian Institute of Technology, Gandhinagar, Ahmedabad-382424, Gujarat, India
}

(Dated: July 22, 2020)

\begin{abstract}
We study a two-species Bose-Einstein condensates confined in quasi-two-dimensional (quasi-2D) optical lattices at finite temperatures, employing the Hartree-Fock-Bogoliubov theory with the Popov approximation. We examine the role of thermal fluctuations on the ground-state density distributions, and the quasiparticle mode evolution. At zero temperature, the geometry of the ground-state in the immiscible domain is side-by-side. Our results show that the thermal fluctuations enhance the miscibility of the condensates, and at a characteristic temperature the system becomes miscible with rotationally symmetric overlapping density profiles. This immiscible-miscible transition is accompanied by a discontinuity in the excitation spectrum, and the low-lying quasiparticle modes such as slosh mode becomes degenerate at the characteristic temperature.
\end{abstract}

PACS numbers: 03.75.Mn, 03.75.Hh, 03.75.Lm, 67.85.Hj

\section{INTRODUCTION}

Ultracold atoms in an optical lattice offer fascinating prospects to investigate many-body quantum physics of strongly correlated systems in a highly controllable environment [1-4]. These systems are recognized as ideal tools to explore new quantum phases [5-7], complex phase transitions [8-11], quantum magnetism [12, 13], quantum information [14] and to simulate transport and magnetic properties of condensed-matter systems [15, 16]. Moreover, the effect of phase separation $[17,18]$, quantum emulsions and coherence properties [19-21], and multicritical behaviour [22, 23] of the mixtures have been explored in the past decade.

Among the various observations made in the two-species Bose-Einstein condensates (TBECs) of ultracold atomic gases, the most remarkable is the phenomenon of phase separation, and it has been a long-standing topic of interest in chemistry and physics. For repulsive on-site interactions, the transition to the phase-separated domain or immiscibility is characterized by the parameter $\Delta=U_{11} U_{22} / U_{12}^{2}-1$, where $U_{11}$ and $U_{22}$ are the intraspecies on-site interactions and $U_{12}$ is the interspecies on-site interaction. When $\Delta<0$, an immiscible phase occurs in which, the atoms of species 1 and 2 have relatively strong repulsion, whereas $\Delta \geqslant 0$ implies a miscible phase [24-26]. It is important to note that the mention criterion is valid at zero temperature for homogeneous systems. The presence of an external trapping potential, however, modifies this condition as the trap introduces an additional energy cost for the species to spatially separate [27]. In experiments, the unique feature of phase separation has been successfully observed in TBECs with harmonic trapping potential [28-30]. Previously, in the context of superfluid Helium at zero temperature, the phase separation of the bosonic mixtures of isotopes of different masses has also been predicted in Refs. [31, 32]. The recent experimental realizations of TBECs in optical lattices, either of two different atomic species [33] or two different hyperfine states of same atomic species [34, 35] provide the motivation to study these systems in detail. In recent works, we have examined the miscible-immiscible transition, and the quasiparticle spectra of the TBECs at zero temperature in quasi-1D [36] and quasi-2D [37] geometries. The finding in the latter work [37], where we had examined the nature of the density profiles in immiscible regime at zero temperature, is of relevance to the present work. In addition, we had shown how the optical lattice potential influences the density profiles in the immiscible domain. The other related study is the ground-state phase diagram, and the effect of filling factor of the TBECs on the phenomenon of phase separation, which were investigated using quantum Monte Carlo simulations [38, 39]. In addition, phase-separation of TBECs at various length scales has been examined using the multi-orbital mean-field theory [40, 41]. Among the full quantum methods the multi-configurational time-dependent Hartree for bosons (MCTDHB) provide a good description of the formation of the interference fringes in the densities during the mixing of condensates $[42,43]$. This method allows the dynamical creation of quantum superposition of states in ultracold Bose gases [44]. In other theoretical studies, the finite temperature properties of TBECs have been explored [45-47]. In continuum or TBECs with harmonic confining potential alone, we have explored the suppression of phase separation due to the presence of the thermal fluctuations [48]. However, a theoretical understanding of the finite temperature effects on the topology and the collective excitations of TBECs in optical lattices is yet to be explored. The Bose-Einstein condensation and hence, the coherence in a system of bosons depends on the interplay between various parameters, such as temperature, interaction strength, confinement, and dimensionality [49]. In particular, in the low-dimensional Bose gases, the coherence can only be maintained across the entire spatial extent at a temperature much below the critical temperature. The coherence property, in experiments, have been studied in recent works [50-54].

With an attention towards this unexplored physics, we study the finite temperature effects of quasi-2D trapped TBECs in optical lattices. In the present work, we address the topological phase transition in the TBECs of two different isotopes of $\mathrm{Rb}$ with temperature as a control parameter in the 
domain $T<T_{c}$, where $T_{c}$ is the critical temperature of either of the species of the mixture. Here, it must be mentioned that in our previous works $[36,37]$, we had investigated the ground-state density, and the quasiparticles with variation in on-site interaction energy at zero temperature. In addition, we have examined the effect of quantum fluctuations on the ground state geometry and collective excitations of the quasi1D TBECs. In the present work, we shall examine the evolution of the quasiparticle modes of TBECs in quasi-2D optical lattices with variation in temperature. For this work, we use Hartree-Fock-Bogoliubov (HFB) formalism with the Popov approximation, and starting from phase-separated domain at zero temperature we increase temperature. We observe that there is an immiscible to miscible transition of the TBEC at a characteristic temperature. This transition is accompanied by a discontinuity in the quasiparticle excitation spectrum, and in addition, some of the modes like the slosh mode become degenerate. We, then, compute the equal-time first-order spatial correlation function which is a measure of the coherence and phase fluctuations present in the system. It describes the off-diagonal long range order which is a defining feature of BEC [55]. This is an important theoretical tool to study the many body effects in atomic physics experiments [56, 57].

This paper is organized as follows. In Sec. II we describe the HFB-Popov formalism, and the numerical techniques used in the present work. The evolution of the quasiparticle modes and the density distributions with the temperature are shown in Sec. III. Finally, our main results are summarized in Sec. IV.

\section{THEORY AND METHODS}

\section{A. HFB-Popov approximation for quasi-2D TBEC}

We consider a TBEC confined in an optical lattice with pancake-shaped configuration of background harmonic trapping potential. Thus, the trapping frequencies satisfy the condition $\omega_{\perp} \ll \omega_{z}$ with $\omega_{x}=\omega_{y}=\omega_{\perp}$. In this system, the excitation energies along the axial direction are high, and the degree of freedom in this direction is frozen. The excitations, both the quantum and thermal fluctuations, are considered only along the radial direction. In the tight-binding approximation (TBA) [58, 59], the Bose-Hubbard (BH) Hamiltonian [60-62] describing this system is

$$
\begin{aligned}
\hat{H}= & \sum_{k=1}^{2}\left[-J_{k} \sum_{\left\langle\xi \xi^{\prime}\right\rangle} \hat{a}_{k \xi}^{\dagger} \hat{a}_{k \xi^{\prime}}+\sum_{\xi}\left(\epsilon_{\xi}^{(k)}-\mu_{k}\right) \hat{a}_{k \xi}^{\dagger} \hat{a}_{k \xi}\right] \\
& +\frac{1}{2} \sum_{k=1, \xi}^{2} U_{k k} \hat{a}_{k \xi}^{\dagger} \hat{a}_{k \xi}^{\dagger} \hat{a}_{k \xi} \hat{a}_{k \xi}+U_{12} \sum_{\xi} \hat{a}_{1 \xi}^{\dagger} \hat{a}_{1 \xi} \hat{a}_{2 \xi}^{\dagger} \hat{a}_{2 \xi},(1)
\end{aligned}
$$

where $k=1,2$ is the species index, $\mu_{k}$ is the chemical potential of the $k$ th species, and $\hat{a}_{k \xi}\left(\hat{a}_{k \xi}^{\dagger}\right)$ is the annihilation (creation) operators of the two different species at $\xi$ th lattice site. The index is such that $\xi \equiv(i, j)$ with $i$ and $j$ as the lattice site index along $x$ and $y$ directions, respectively. The summation index $\left\langle\xi \xi^{\prime}\right\rangle$ represents the sum over nearest-neighbour to the $\xi$ th site. The TBA is valid when the depth of the lattice potential is much larger than the chemical potential $V_{0} \gg \mu_{k}$, the BH Hamiltonian then describes the system when the bosonic atoms occupy the lowest energy band. A detailed derivation of the BH Hamiltonian is given in our previous works [36, 37]. In the BH Hamiltonian, $J_{k}$ are the tunneling matrix elements, $\epsilon_{\xi}^{(k)}$ is the offset energy arising due to background harmonic potential, and $U_{k k}\left(U_{12}\right)$ are the intraspecies (interspecies) interaction strengths. In the present work all the interaction strengths are considered to be repulsive, that is, $U_{k k}, U_{12}>0$.

In the weakly interacting regime, under the Bogoliubov approximation [63, 64], the annihilation operators at each lattice site can be written as $\hat{a}_{1 \xi}=\left(c_{\xi}+\hat{\varphi}_{1 \xi}\right) e^{-i \mu_{1} t / \hbar}$, $\hat{a}_{2 \xi}=\left(d_{\xi}+\hat{\varphi}_{2 \xi}\right) e^{-i \mu_{2} t / \hbar}$, where $c_{\xi}$ and $d_{\xi}$ are the complex amplitudes describing the condensate phase of each of the species. The operators $\hat{\varphi}_{1 \xi}$ and $\hat{\varphi}_{2 \xi}$ representing the quantum or thermal fluctuation part of the field operators. Further more, we consider the system in the superfluid domain where the mean-field description is applicable, and accordingly, the parameters satisfy the condition $U / J \ll 16.7$ [65-67]. In this domain, the equation of motion of the condensate in optical lattice with TBA is reduced to discrete nonlinear Schrödinger equation (DNLSE). However, in the Mott-insulator phase, $U / J \geqslant 16.7$, the mean-field description breaks down, and a full quantum description is required [68-70]. From the equation of motion of the field operators with the Bogoliubov approximation, the equilibrium properties of a TBEC is governed by the coupled generalized DNLSEs

$\mu_{1} c_{\xi}=-J_{1} \sum_{\xi^{\prime}} c_{\xi^{\prime}}+\left[\epsilon_{\xi}^{(1)}+U_{11}\left(n_{1 \xi}^{c}+2 \tilde{n}_{1 \xi}\right)+U_{12} n_{2 \xi}\right] c_{\xi}$,

$\mu_{2} d_{\xi}=-J_{2} \sum_{\xi^{\prime}} d_{\xi^{\prime}}+\left[\epsilon_{\xi}^{(2)}+U_{22}\left(n_{2 \xi}^{c}+2 \tilde{n}_{2 \xi}\right)+U_{12} n_{1 \xi}\right] d_{\xi}$,

where $n_{1 \xi}^{c}=\left|c_{\xi}\right|^{2}$ and $n_{2 \xi}^{c}=\left|d_{\xi}\right|^{2}, \tilde{n}_{k \xi}=\left\langle\hat{\varphi}_{k \xi}^{\dagger} \hat{\varphi}_{k \xi}\right\rangle$, and $n_{k \xi}=n_{k \xi}^{c}+\tilde{n}_{k \xi}$ are the condensate, noncondensate, and total density of the species, respectively. The fluctuation operators are defined in terms of the quasiparticles through the Bogoliubov transformation

$$
\hat{\varphi}_{k \xi}=\sum_{l}\left[u_{k \xi}^{l} \hat{\alpha}_{l} e^{-i \omega_{l} t}-v_{k \xi}^{* l} \hat{\alpha}_{l}^{\dagger} e^{i \omega_{l} t}\right]
$$

where $\hat{\alpha}_{l}\left(\hat{\alpha}_{l}^{\dagger}\right)$ are the quasiparticle annihilation (creation) operators, which satisfy the Bose commutation relations, $l$ is the quasiparticle mode index, $u_{k \xi}^{l}$ and $v_{k \xi}^{l}$ are the quasiparticle amplitudes for the $k$ th species, and $\omega_{l}=E_{l} / \hbar$ is the frequency of the $l$ th quasiparticle mode with $E_{l}$ as the mode excitation energy.

Using the Bogoliubov transformation, we obtain the fol- 
lowing HFB-Popov equations [37]:

$$
\begin{aligned}
E_{l} u_{1, \xi}^{l}= & -J_{1}\left(u_{1, \xi-1}^{l}+u_{1, \xi+1}^{l}\right)+\mathcal{U}_{1} u_{1, \xi}^{l}-U_{11} c_{\xi}^{2} v_{1, \xi}^{l} \\
& +U_{12} c_{\xi}\left(d_{\xi}^{*} u_{2, \xi}^{l}-d_{\xi} v_{2, \xi}^{l}\right), \\
E_{l} v_{1, \xi}^{l}= & J_{1}\left(v_{1, \xi-1}^{l}+v_{1, \xi+1}^{l}\right)+\underline{\mathcal{U}}_{1} v_{1, \xi}^{l}+U_{11} c_{\xi}^{* 2} u_{1, \xi}^{l} \\
& -U_{12} c_{\xi}^{*}\left(d_{\xi} v_{2, \xi}^{l}-d_{\xi}^{*} u_{2, \xi}^{l}\right), \\
E_{l} u_{2, \xi}^{l}= & -J_{2}\left(u_{2, \xi-1}^{l}+u_{2, \xi+1}^{l}\right)+\mathcal{U}_{2} u_{2, \xi}^{l}-U_{22} d_{\xi}^{2} v_{2, \xi}^{l} \\
& +U_{12} d_{\xi}\left(c_{\xi}^{*} u_{1, \xi}^{l}-c_{\xi} v_{1, \xi}^{l}\right), \\
E_{l} v_{2, \xi}^{l}= & J_{2}\left(v_{2, \xi-1}^{l}+v_{2, \xi+1}^{l}\right)+\underline{\mathcal{U}}_{2} v_{2, \xi}^{l}+U_{22} d_{\xi}^{* 2} u_{2, \xi}^{l} \\
& -U_{12} d_{\xi}^{*}\left(c_{\xi} v_{1, \xi}^{l}-c_{\xi}^{*} u_{1, \xi}^{l}\right),
\end{aligned}
$$

where $\mathcal{U}_{1}=2 U_{11}\left(n_{1 \xi}^{c}+\tilde{n}_{1 \xi}\right)+U_{12}\left(n_{2 \xi}^{c}+\tilde{n}_{2 \xi}\right)+\left(\epsilon_{\xi}^{(1)}-\right.$ $\left.\mu_{1}\right), \mathcal{U}_{2}=2 U_{22}\left(n_{2 \xi}^{c}+\tilde{n}_{2 \xi}\right)+U_{12}\left(n_{1 \xi}^{c}+\tilde{n}_{1 \xi}\right)+\left(\epsilon_{\xi}^{(2)}-\mu_{2}\right)$ with $\underline{\mathcal{U}}_{k}=-\mathcal{U}_{k}$. To solve the above eigenvalue equation, we use a basis set of on-site Gaussian wave functions, and define the quasiparticle amplitude as linear combination of the basis functions. The condensate and noncondensate densities are then computed through the self-consistent solution of Eqs. (2) and (4). The noncondensate atomic density at the $\xi$ th lattice site is

$$
\tilde{n}_{k \xi}=\sum_{l}\left[\left(\left|u_{k \xi}^{l}\right|^{2}+\left|v_{k \xi}^{l}\right|^{2}\right) N_{0}\left(E_{l}\right)+\left|v_{k \xi}^{l}\right|^{2}\right],
$$

where $N_{0}\left(E_{l}\right)=\left(e^{\beta E_{l}}-1\right)^{-1}$ with $\beta=\left(k_{B} T\right)^{-1}$ is the Bose-Einstein distribution factor of the $l$ th quasiparticle mode with energy $E_{l}$ at temperature $T$. The last term in $\tilde{n}_{k \xi}$ is independent of the temperature, and hence, represents the quantum fluctuations of the system. To examine the role of temperature we define the miscibility of the condensates in terms of the overlap integral

$$
\Lambda=\frac{\left[\int n_{1}(\mathbf{r}) n_{2}(\mathbf{r}) d \mathbf{r}\right]^{2}}{\left[\int n_{1}^{2}(\mathbf{r}) d \mathbf{r}\right]\left[\int n_{2}^{2}(\mathbf{r}) d \mathbf{r}\right]} .
$$

Here, $n_{k}(\mathbf{r})$ is the total density of $k$ th condensate at position $\mathbf{r} \equiv(x, y)$. If the two condensates of the TBEC have complete overlap to each other then the system is in miscible phase with $\Lambda=1$, whereas for the completely phaseseparated case $\Lambda=0$. Using $\Lambda$ as a measure we identify the miscible and immiscible domain as a function of the temperature. As we use the coupled DNLSEs to describe the TBEC, our study is valid deep within the superfluid domain, and the mean-field description would begin to deviate from the true results near the superfluid-Mott-insulator phase transition. In this regime a full quantum description [70] would be the appropriate method, and the same applies to probing the nature of the quantum phase transition [71-74]. It is well established that for some parameter regimes, TBECs in optical lattices can either be superfluid phase of both the species, or superfluid phase of one species coexisting with the Mott insulator phase of the other [75-78].

\section{B. Field-field correlation function}

To define a measure of the coherence in the condensate we introduce the first-order correlation function $g_{k}^{(1)}\left(\mathbf{r}, \mathbf{r}^{\prime}\right)$, which can be expressed as expectations of product of field operators at different positions and times [79-82]. These are normalized to obtain unit modulus in the case of perfect coherence or a system consisting of only condensate atoms. Here, we restrict ourselves to ordered spatial correlation functions at a fixed and equal time. In terms of the quantum Bose field operator $\hat{\Psi}_{k}$ the first-order spatial correlation function is

$$
g_{k}^{(1)}\left(\mathbf{r}, \mathbf{r}^{\prime}\right)=\frac{\left\langle\hat{\Psi}_{k}^{\dagger}(\mathbf{r}) \hat{\Psi}_{k}\left(\mathbf{r}^{\prime}\right)\right\rangle}{\sqrt{\left\langle\hat{\Psi}_{k}^{\dagger}(\mathbf{r}) \hat{\Psi}_{k}(\mathbf{r})\right\rangle\left\langle\hat{\Psi}_{k}^{\dagger}\left(\mathbf{r}^{\prime}\right) \hat{\Psi}_{k}\left(\mathbf{r}^{\prime}\right)\right\rangle}},
$$

where $\langle\cdots\rangle$ represents thermal average. It is important to note that the local first-order correlation function is equal to the density, i.e. $g_{k}^{(1)}(\mathbf{r}, \mathbf{r})=n_{k}(\mathbf{r})$. The expression of $g_{k}^{(1)}\left(\mathbf{r}, \mathbf{r}^{\prime}\right)$ can also be written in terms of condensate, and noncondensate density correlations as

$$
g_{k}^{(1)}\left(\mathbf{r}, \mathbf{r}^{\prime}\right)=\frac{n_{k}^{c}\left(\mathbf{r}, \mathbf{r}^{\prime}\right)+\tilde{n}_{k}\left(\mathbf{r}, \mathbf{r}^{\prime}\right)}{\sqrt{n_{k}(\mathbf{r}) n_{k}\left(\mathbf{r}^{\prime}\right)}}
$$

where

$$
\begin{aligned}
n_{k}^{c}\left(\mathbf{r}, \mathbf{r}^{\prime}\right)= & \psi_{k}^{*}(\mathbf{r}) \psi_{k}\left(\mathbf{r}^{\prime}\right), \\
\tilde{n}_{k}\left(\mathbf{r}, \mathbf{r}^{\prime}\right)= & \sum_{l}\left[\left\{u_{k}^{* l}(\mathbf{r}) u_{k}^{l}\left(\mathbf{r}^{\prime}\right)+v_{k}^{* l}(\mathbf{r}) v_{k}^{l}\left(\mathbf{r}^{\prime}\right)\right\} N_{0}\left(E_{l}\right)\right. \\
& \left.+v_{k}^{* l}(\mathbf{r}) v_{k}^{l}\left(\mathbf{r}^{\prime}\right)\right] \\
n_{k}(\mathbf{r})= & n_{k}^{c}(\mathbf{r})+\tilde{n}_{k}(\mathbf{r})
\end{aligned}
$$

are the condensate density correlation, noncondensate density correlation, and total density of the $k$ th species, respectively. In the above expressions, $n_{k}^{c}\left(\mathbf{r}, \mathbf{r}^{\prime}\right)$ and $\tilde{n}_{k}\left(\mathbf{r}, \mathbf{r}^{\prime}\right)$ are obtained by expanding the complex amplitudes $\left(c_{\xi}, d_{\xi}\right)$ and the quasiparticle amplitudes $\left(u_{k, \xi}^{l}, v_{k, \xi}^{l}\right)$ in the localized Gaussian basis. At $T=0 \mathrm{~K}$, the entire condensate cloud has complete coherence, and therefore $g_{k}^{(1)}=1$ within the condensate region. In TBECs, the transition from phase-separated to the miscible domain at $T \neq 0$ has characteristic signatures in the spatial structure of $g_{k}^{(1)}\left(\mathbf{r}, \mathbf{r}^{\prime}\right)$.

\section{Numerical methods}

To solve the coupled DNLSEs, Eqs. (2), we scale and rewrite the equations in the dimensionless form. For this we choose the characteristic length scale as the lattice constant $a=\lambda_{L} / 2$ with $\lambda_{L}$ as the wavelength of the laser which creates the lattice potential. Similarly, the recoil energy $E_{R}=\hbar^{2} k_{L}^{2} / 2 m$ with $m$ is the atomic mass of the species and $k_{L}=2 \pi / \lambda_{L}$ is chosen as the energy scale of the system. We use fourth-order Runge-Kutta method to solve these equations for zero as well as finite temperatures. To initiate the iterative steps to solve the equations an appropriate initial 
guess value of $c_{\xi}$ and $d_{\xi}$ are chosen. For the present work we chose the values corresponding to the side-by-side profile as it gives quasiparticle energies which are real and not complex. This is important as this shows that the solution we obtain is a stable one, and not a metastable one. The stationary ground-state wave-function of the TBEC is obtained through imaginary-time propagation. In the tight-binding limit, the width of the orthonormalized Gaussian basis functions localized at each lattice site is $0.3 a$. Furthermore, to study the quasiparticle excitation spectrum, we cast Eqs. (4) as matrix eigenvalue equation, and diagonalize the matrix using the routine ZGEEV from the LAPACK library [83]. For finite temperature computations, to take into account the thermal fluctuations, we solve the coupled equations Eqs. (2) and Eqs. (4) self-consistently. The solution of the DNLSEs is iterated until it satisfies the convergence criteria in terms of the number of condensate and noncondensate atoms. In general, the convergence is not smooth, and most of the time we encounter severe oscillations in the number of atoms. To remedy these oscillations and attain convergence, we damp the solution using the successive over- (under-) relaxation technique while updating the condensate (noncondensate) atoms. Thus, the new solutions after an iteration cycle (IC) are

$$
\begin{array}{r}
c_{\xi, \mathrm{IC}}^{\text {new }}=r^{\mathrm{ov}} c_{\xi, \mathrm{IC}}+\left(1-r^{\mathrm{ov}}\right) c_{\xi, \mathrm{IC}-1}, \\
d_{\xi, \mathrm{IC}}^{\text {new }}=r^{\mathrm{ov}} d_{\xi, \mathrm{IC}}+\left(1-r^{\mathrm{ov}}\right) d_{\xi, \mathrm{IC}-1}, \\
\tilde{n}_{k \xi, \mathrm{IC}}^{\text {new }}=r^{\mathrm{un}} \tilde{n}_{k \xi, \mathrm{IC}}+\left(1-r^{\mathrm{un}}\right) \tilde{n}_{k \xi, \mathrm{IC}-1},
\end{array}
$$

where $r^{\text {ov }}>1\left(r^{\text {un }}<1\right)$ is the over (under) relaxation parameter. The choice of $r^{\mathrm{ov}}$ and $r^{\mathrm{un}}$ depend on the temperature and interaction parameters. In general, our observation is that the oscillations are more prominent at higher temperatures, and hence, lower values of $r^{\text {ov }}$ and $r^{\text {un }}$ must be chosen. This in turn implies that it takes larger number of iterations to get converged solutions at higher temperatures.

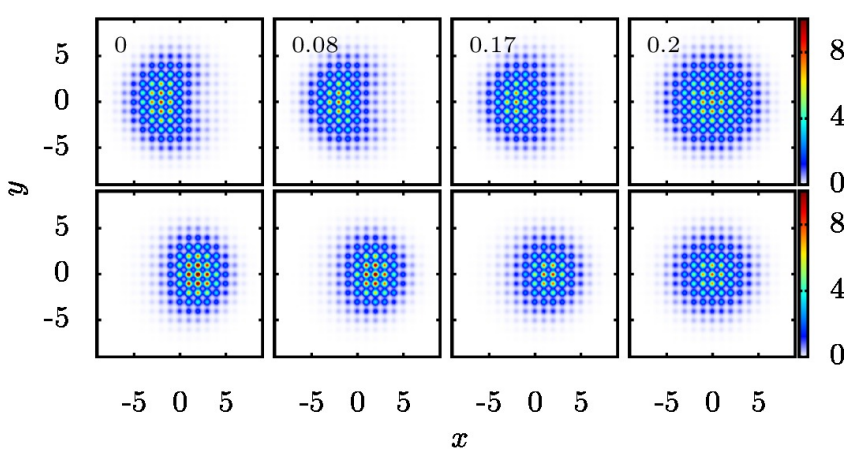

FIG. 1. The density distribution for the condensate atoms of ${ }^{87} \mathrm{Rb}$ ${ }_{-}^{85} \mathrm{Rb}$ TBEC as a function of the temperature $T / T_{c}$. The density profiles of the ${ }^{87} \mathrm{Rb}$ (upper panel) and ${ }^{85} \mathrm{Rb}$ species (lower panel) are shown for $T / T_{c}=0,0.08,0.17$, and 0.2 . In the phase-separated domain, the condensate density has side-by-side geometry at zero temperature, and as temperature is increased, there is a transition to miscible domain or the densities completely overlap at $T_{\mathrm{ch}}=0.185 T_{c}$. Here $x$ and $y$ are measured in units of the lattice constant $a$.

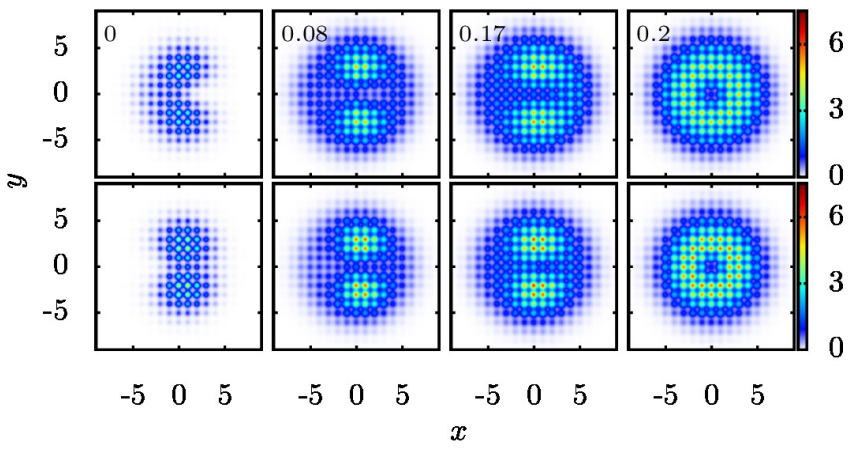

FIG. 2. The density distribution for the noncondensate atoms of ${ }^{87} \mathrm{Rb}$ ${ }_{-}^{85} \mathrm{Rb}$ TBEC as a function of the temperature $T / T_{c}$. The noncondensate density of the ${ }^{87} \mathrm{Rb}$ (upper panel) and ${ }^{85} \mathrm{Rb}$ species (lower panel) are shown for $T / T_{c}=0,0.08,0.17$, and 0.2 . The noncondensate atoms which are localized at the edges, acquire rotational symmetry in the miscible phase, which happens at $T_{\mathrm{ch}}=0.185 T_{c}$ as the temperature is increased. Here $x$ and $y$ are measured in units of the lattice constant $a$.

\section{RESULTS AND DISCUSSIONS}

To examine the effects of thermal fluctuations on the quasiparticle spectra we consider the ${ }^{87} \mathrm{Rb}-{ }^{85} \mathrm{Rb}$ TBEC with ${ }^{87} \mathrm{Rb}$ labeled as species 1 and ${ }^{85} \mathrm{Rb}$ labeled as species 2 . The radial trapping frequencies of the harmonic potential are $\omega_{x}=$ $\omega_{y}=\omega_{\perp}=2 \pi \times 50 \mathrm{~Hz}$ with the anisotropy parameter $\omega_{z} / \omega_{\perp}=20.33$, and these parameters are chosen based on the experimental work of Gadway and collaborators [34] on the TBEC of two hyperfine states of ${ }^{87} \mathrm{Rb}$ in optical lattices. It is important to note that we consider equal background trapping potential for species 1 and 2 . We emphasized here that, the results are equally applicable to the case of the TBEC consisting of two hyperfine states of ${ }^{87} \mathrm{Rb}$, however, we have chosen ${ }^{87} \mathrm{Rb}-{ }^{85} \mathrm{Rb}$ to highlight that the small mass difference have no influence on the geometry of the ground state. The laser wavelength used to create the $2 \mathrm{D}$ lattice potential and the lattice depth are $\lambda_{L}=1064 \mathrm{~nm}$ and $V_{0}=5 E_{R}$, respectively. We then take the total number of atoms as $N_{1}=N_{2}=100$ confined in a $40 \times 40$ quasi-2D lattice system. It must be mentioned that the number of lattice sites considered much larger than the spatial extent of the condensate cloud. Albeit the computations require longer time with the larger lattice size, we chose it to ensure that the spatial extent of the thermal component is confined well within the lattice considered. The tunneling matrix elements are $J_{1}=0.66 E_{R}$ and $J_{2}=0.71 E_{R}$, which correspond to an optical lattice potential with a depth of $5 E_{R}$. The intraspecies and interspecies on-site interactions are set as $U_{11}=0.07 E_{R}, U_{22}=0.02 E_{R}$ and $U_{12}=0.15 E_{R}$, respectively. For this set of parameters the ground-state density distribution of ${ }^{87} \mathrm{Rb}-{ }^{85} \mathrm{Rb}$ TBEC is phase-separated with side-by-side geometry. This is a symmetry-broken profile where one species is placed to the left and other to the right of the trap center along $y$-axis. The evolution of the ground state from miscible to the side-by-side density profile due to decrease in the $U_{22}$ is reported in our previous work [37]. In the present work, we demonstrate the role of temperature in 
the phase-separated domain of the binary condensate.

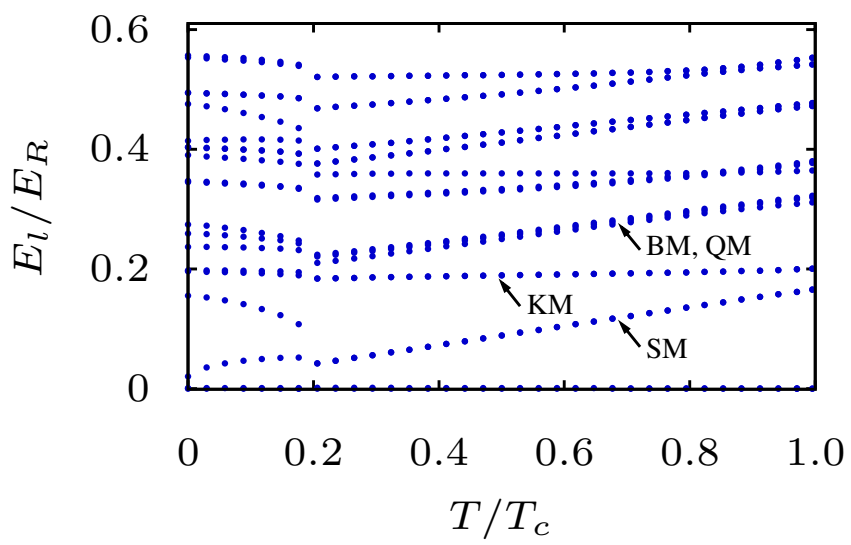

FIG. 3. The evolution of the excitation energies of the low-lying quasiparticle modes as a function of the temperature in ${ }^{87} \mathrm{Rb}-{ }^{85} \mathrm{Rb}$ TBEC. The slosh and some of the other higher energy modes become degenerate at $T_{\mathrm{ch}}=0.185 T_{c}$, where the density distribution is transformed from phase-separated to the miscible profile. In the plot, the slosh mode (SM), Kohn mode (KM), breathing mode (BM), and quadrupole mode $(\mathrm{QM})$ are marked by the black arrows. Here, the excitation energy $E_{l}$, and the temperature $T$ are scaled with respect to the recoil energy $E_{R}$, and the critical temperature $T_{c}$ of the ${ }^{87} \mathrm{Rb}$ species, respectively.

\section{A. Zero temperature}

At zero temperature, in the phase-separated domain, the energetically preferable ground state of TBEC is the side-byside geometry, which is reported in our previous work [37]. Unlike in one-dimensional system [36] in quasi-2D system the presence of the quantum fluctuations does not alter the ground state. For the parameters chosen ${ }^{87} \mathrm{Rb}-{ }^{85} \mathrm{Rb}$ TBEC is phase separated, and the overlap integral has the value $\Lambda=0.10$. The density distributions of the condensate and noncondensate atoms of the two species at zero temperature is shown in Fig. 1 and Fig. 2. This is a symmetry broken side-by-side geometry with noncondensate atoms more localized at the edges of the condensate along $y$-axis.

\section{B. Finite temperatures}

At $T \neq 0$, in addition to the quantum fluctuations, which are present at the zero temperature, the thermal cloud also contribute to the noncondensate density. As shown in Figs. 1 and 2 , at $T / T_{c}=0.08$, the condensate density profiles of both the species begin to overlap, or in other words, the two species are partly miscible. This is also evident from the value of $\Lambda=0.16$, which shows a marginal increase compared to the value of 0.10 at zero temperature. In the figures, the temperature is defined in units of the critical temperature $T_{c}$ of ${ }^{87} \mathrm{Rb}$ atoms, which for the parameters considered is $338 \mathrm{nK}$ based on our finite temperature computations. This value of

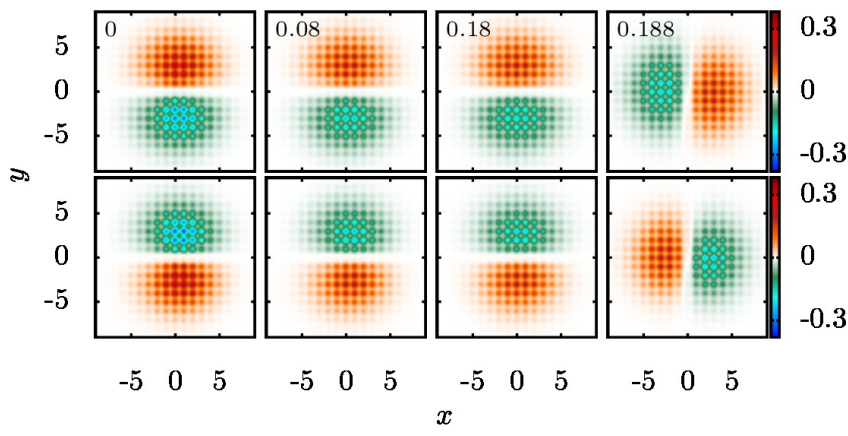

FIG. 4. The quasiparticle mode function of the first excited mode (slosh mode) as a function of the temperature for the ${ }^{87} \mathrm{Rb}-{ }^{85} \mathrm{Rb}$ TBEC. The mode function corresponding to the ${ }^{87} \mathrm{Rb}$ and ${ }^{85} \mathrm{Rb}$ species are shown in the upper and lower panel, respectively. The slosh mode is an out-of-phase mode where the density flow of the two species are in opposite directions. As the TBEC acquires rotational symmetry at $T_{\mathrm{ch}}=0.185 T_{c}$, the slosh mode is rotated by an angle $\pi / 2$ for $T / T_{c} \geqslant 0.185$. The value of $T / T_{c}$ is shown at the upper left corner of each plot in the upper panel. The spatial coordinate $x$ and $y$ are in units of the lattice constant $a$.

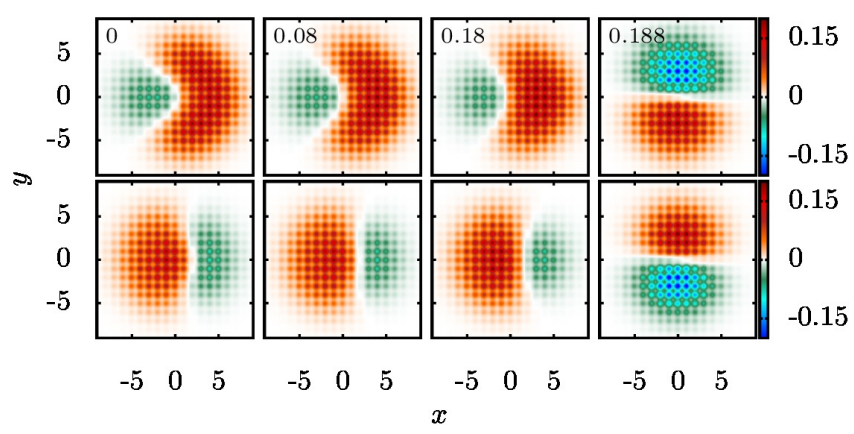

FIG. 5. The quasiparticle mode function corresponding to the second excited mode (slosh mode), which becomes degenerate with the first excited mode for $T / T_{c} \geqslant 0.185$. The mode function of the ${ }^{87} \mathrm{Rb}$ and ${ }^{85} \mathrm{Rb}$ species are shown in the upper and lower panel, respectively. The value of $T / T_{c}$ is shown at the upper left corner of each plot in the upper panel. Here $x$ and $y$ are in units of the lattice constant $a$.

$T_{c}$ is consistent with the analytic expression for ideal Bose gas in optical lattices [84]

$$
T_{c}=\frac{m \omega^{2} a^{2}}{2 \pi k_{B}}\left[\frac{N_{k}}{\zeta(3 / 2)}\right]^{2 / 3}
$$

where $\omega$ is the geometric mean of the three oscillator frequencies, $N_{k}$ is the number of atoms of $k$ th species and $\zeta(3 / 2)=2.612$ is the Riemann zeta function. In the presence of the harmonic confinement, the repulsive interatomic interaction reduce the density at the trap center and hence decreases $T_{c}$ [84]. Upon further increase in temperature, at $T / T_{c}=0.18, \Lambda=0.36$, this indicates an increase in the miscibility of the two species. Another important feature at $T / T_{c}=0.08$ and 0.18 is the localization of the noncondensate atoms at the interface. This is due to repulsion from the condensate atoms, and lower thermal energy which is insufficient 


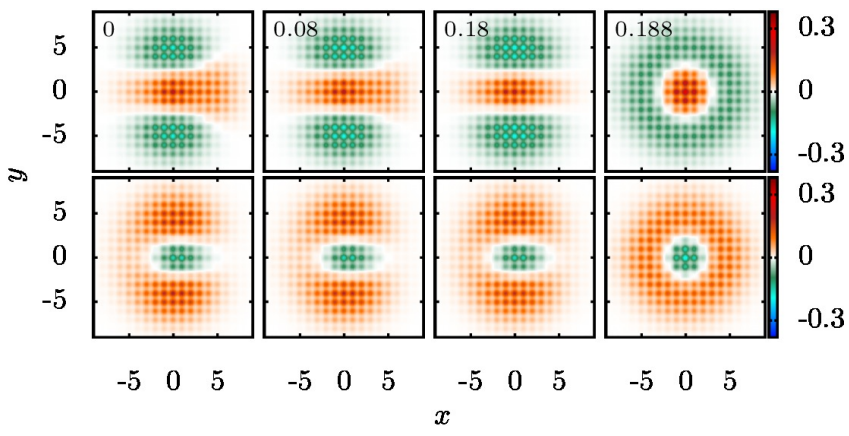

FIG. 6. The quasiparticle mode function corresponding to the interface mode in the phase-separated domain of ${ }^{87} \mathrm{Rb}-{ }^{85} \mathrm{Rb}$ TBEC as a function of the temperature. This is an out-of-phase mode and the mode function is more prominent at the interface region between the condensates. For $T / T_{c} \geqslant 0.185$, when the TBEC acquires the rotational symmetry, this mode is transformed into the out-of-phase breathing mode, where the mode functions are radially symmetric. The value of $T / T_{c}$ is shown at the upper left corner of each plot in the upper panel. Here $x$ and $y$ are in units of the lattice constant $a$.

to overcome this repulsion energy. The transition to miscible domain occurs when the temperature exceeds the characteristic temperature

$$
T_{\mathrm{ch}} \approx \frac{\sqrt{n_{1 \max } n_{2 \max }} U_{12}}{k_{B}},
$$

where $n_{k \max }$ is the maximum density of the $k$ th species. At higher temperatures, the extent of overlap between the condensate density profiles increases, and TBEC is completely miscible at $T_{\mathrm{ch}}=0.185 T_{c} \approx 63 \mathrm{nK}$. This is reflected in the value of $\Lambda=0.95$, and the condensate as well as the noncondensate densities acquire rotational symmetry. The $T_{\mathrm{ch}}$ at which this transition occurs correspond to the thermal energy $k_{B} T_{\mathrm{ch}}=0.72 E_{R}$, which is comparable to the the interspecies interaction energy of $0.66 E_{R}$. Albeit, we discuss in detail the results for the parameters mentioned earlier, we find similar trends in the immiscible-miscible transition for different values of $J$ 's and $U$ 's. As to be expected the only change is that the $T_{\text {ch }}$ is lowered with higher $J$. This is due to the higher kinetic energy associated with higher $J$, and hence the atoms require less thermal energy to overcome the interspecies repulsion energy for transition to the miscible phase. In terms of the interaction energies, the lower value of $U_{k k}$ and higher value of $U_{12}$ increase the $T_{\mathrm{ch}}$ of the TBEC.

The transition from the phase-separated into miscible domain can further be examined from the evolution of the quasiparticle modes as a function of the temperature. The evolution of the few low-lying mode energies with temperature is shown in Fig. 3, where the temperature is defined in units of $T_{c}$. It is evident from the figure that there are mode energy bifurcations with the increase in the temperature. These are associated with the restoration of rotational symmetry when the TBEC is rendered miscible through an increase in temperature.

As to be expected the two lowest energy modes are the zero energy or the Goldstone modes, which are the result of the spontaneous symmetry breaking associated with the condensation. In the phase-separated domain, these modes correspond to one each for each of the species. The first two excited modes are the non-degenerate Kohn or slosh modes of the two species, and these remain non-degenerate in the domain $T<T_{\mathrm{ch}}$. The structure of these modes are shown in Figs. 4 and 5. When $T \geqslant T_{\text {ch }}$ as the TBEC acquires a rotational symmetry, the slosh modes becomes degenerate with $\pi / 2$ rotation. A key feature in the quasiparticle mode evolution is that the energy of all the out-of-phase mode increases for $T \geqslant T_{\text {ch }}$, whereas all the in-phase mode remains steady. Here, out-of-phase and in-phase means that the amplitudes $u_{1}$ and $u_{2}$ of a quasiparticle are of different and same phases, respectively. Among the low-energy modes, the Kohn mode is in phase whereas the breathing and quadrupole modes are out of phase in nature. One unique feature of TBEC in the immiscible phase is the presence of interface modes, these have amplitudes prominent around the interface region. The existence of these modes is reported in our previous work [37], and were investigated in other works $[85,86]$ for TBECs confined in harmonic potential alone at zero temperature. As an example, one of the low-energy interface modes is shown in Fig. 6. It is evident from the figure that the mode is out of phase in nature, and it is transformed into breathing mode in the miscible domain when $T \geqslant T_{\mathrm{ch}}$. In the miscible domain, the breathing mode becomes degenerate with the quadrupole mode, and gains energy. The quasiparticles of the miscible domain have well-defined azimuthal quantum number, and modes undergo rotations as $T$ is further increased. To gain

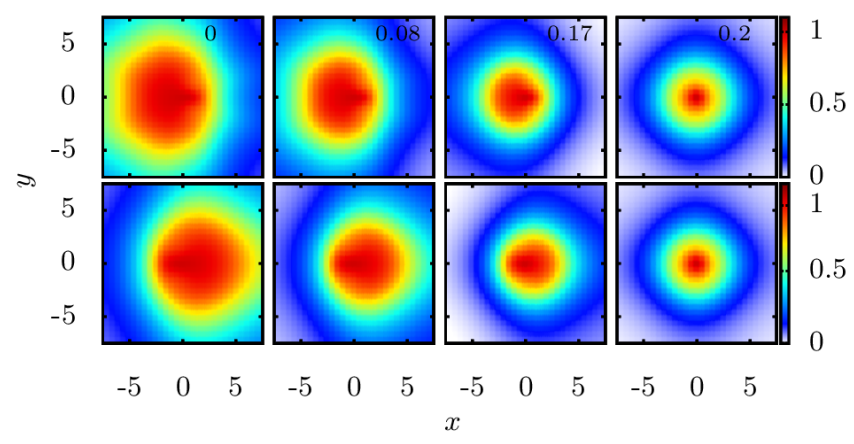

FIG. 7. The normalized first-order spatial correlation function $g_{k}^{(1)}(0, \mathbf{r})$ for ${ }^{87} \mathrm{Rb}$ (upper panel) and ${ }^{85} \mathrm{Rb}$ (lower panel) species at $T / T_{c}=0,0.08,0.17$, and 0.2 . Here $x$ and $y$ are measured in units of the lattice constant $a$.

additional insights on the immiscible-miscible transition, we consider other TBECs. In particular, we consider $\mathrm{Rb}-\mathrm{Cs}$ and $\mathrm{Rb}-\mathrm{K}$ TBECs confined in quasi-2D optical lattices. The details of the parameters chosen and discussion are given in the appendix. Starting from the immiscible domain we analyze the ground state, and the quasiparticle mode evolution with increase in the temperature. Based on the results we observe that the trends in the evolution of the low-lying quasiparticle modes with temperature is qualitatively similar to the ${ }^{87} \mathrm{Rb}$ ${ }^{85} \mathrm{Rb}$ TBEC. The condensate density profiles also exhibit the same trend of transformation from immiscible side-by-side geometry to the rotationally symmetric miscible profile. As 
to be expected, the value of the $T_{\mathrm{ch}}$ depends on the mass ratio, this is due to the mass dependence of interaction energy. In particular, for Rb-Cs and Rb-K TBECs, $T_{\text {ch }}$ are $0.62 T_{c}$, and $0.53 T_{c}$, respectively. The thermal energies corresponding to these temperatures are $2.15 E_{R}$ and $2.80 E_{R}$, respectively. These are comparable to the interaction energies of the TBECs, which are $1.97 E_{R}$ and $2.84 E_{R}$, respectively. Here $T_{c}$ is the critical temperature of the condensation for the species with lower value. In addition to the atomic mass of the condensates, as mentioned earlier, the immiscible-miscible transition also depends on the lattice parameters $U$ 's and $J$ 's. For these two TBECs also we have examined the density distributions with variation in $U$ 's and $J$ 's parameters. We find similar trends in the value of the $T_{\mathrm{ch}}$ as in ${ }^{87} \mathrm{Rb}-{ }^{85} \mathrm{Rb}$ TBEC. That is, decrease in $T_{\text {ch }}$ with increase in $J$, and increase with lower and higher values of $U_{k k}$ and $U_{12}$, respectively.

To investigate the spatial coherence of TBEC at equilibrium, we examine the trends in $g_{k}^{(1)}(0, \mathbf{r})$ defined earlier in Eq. (8), and are shown in Fig. 7 for various temperatures. As mentioned earlier, at zero temperature, $n_{k}(\mathbf{r}) \approx n_{k}^{c}(\mathbf{r})$ have complete phase coherence, and therefore, $g_{k}^{(1)}=1$ within the spatial extent of the condensates, this is shown in Fig. 7. At zero temperature or in the limit $\tilde{n}_{k} \equiv 0$ the correlation function, Eq. (8), resemble a Heaviside function, and the negligible contribution from the quantum fluctuations smooth out the sharp edges as $g_{k}^{(1)}$ drops to zero. More importantly, in the numerical computations this cause a loss of numerical accuracy as it involves division of two small numbers in Eq. (8) [87]. However at finite temperature the presence of the noncondensate atoms modify the nature of the spatial coherence present in the system. The decay rate of the correlation function increases with the temperature, and this is evident from Fig. 7, which shows $g_{k}^{(1)}(0, \mathbf{r})$ at $T / T_{c}=0.08,0.17$, and 0.2 . In addition to this, the transition from phase-separated to the miscible TBEC is also reflected in the decay trends of $g_{k}^{(1)}(0, \mathbf{r})$.

\section{CONCLUSIONS}

We have examined the finite temperature effects on the phenomenon of phase separation in TBECs confined in quasi$2 \mathrm{D}$ optical lattices. As temperature is increased the phaseseparated side-by-side ground state geometry is transformed into miscible phase. For the case of TBEC comprising of ${ }^{87} \mathrm{Rb}$ and ${ }^{85} \mathrm{Rb}$, the transformation occurs at the characteristic temperature. This demonstrates the importance of thermal fluctuations which can make TBECs miscible. Based on the present work, in general, the TBEC undergoes transition to miscible phase at a characteristic temperature $T_{\mathrm{ch}}$. This corresponds to the temperature at which the thermal energy overcomes the interspecies repulsion energy $\sqrt{n_{1 \max } n_{2 \max }} U_{12}$. The other key observation is that the transition from phase-separated domain to miscible domain is associated with a change in the nature of the quasiparticle energies. The low-lying out-of-phase mode, in particular, the slosh mode becomes degenerate and increase in energy. On the other hand, the in-phase mode, such as Kohn mode, remains steady as temperature $\left(T<T_{c}\right)$ is increased. The interface modes, which are unique to the phase-separated domain, in addition to change in energy are geometrically transformed into rotationally symmetric breathing modes in the miscible domain. The temperature driven immiscible to the miscible transition is also evident in the profile of the correlation functions.

\section{ACKNOWLEDGMENTS}

We thank Arko Roy, S. Gautam, S. Bandyopadhyay and R. Bai for useful discussions. The results presented in the paper are based on the computations using Vikram-100, the 100TFLOP HPC Cluster at Physical Research Laboratory, Ahmedabad, India.

\section{APPENDIX}

Here, we provide brief descriptions of the computations pertaining to the $\mathrm{Rb}-\mathrm{Cs}$ and $\mathrm{Rb}-\mathrm{K}$ TBECs confined in quasi2D optical lattices.

\section{A. ${ }^{87} \mathbf{R b}-{ }^{133} \mathbf{C s}$ TBEC}

We consider ${ }^{87} \mathrm{Rb}-{ }^{133} \mathrm{Cs}$ TBEC containing 100 atoms of each species confined in a $40 \times 40$ quasi-2D optical lattices with the wavelength of the laser beams as $1064 \mathrm{~nm}$. The lower number of atoms are chosen to improve the convergence of finite temperature computations, and at the same time it is sufficient to provide a good description of the superfluid phase of the TBECs. The radial trapping frequencies of the external harmonic trapping potential are $\omega_{x}=\omega_{y}=\omega_{\perp}=2 \pi \times 50$ $\mathrm{Hz}$ with the anisotropy parameter as 20.33 [34]. The tunneling matrix elements are $J_{1}=0.66 E_{R}$ and $J_{2}=1.70 E_{R}$ corresponding to the depth of optical lattice $V_{0}=5 E_{R}$. The lattice depth are considered such that the tight-binding limit, $V_{0} \gg \mu_{k}$, is valid. The large difference in the values of $J_{k}$ is due to the large mass difference between the atoms of the two species. The intraspecies and interspecies on-site interactions considered are $U_{11}=0.96 E_{R}, U_{22}=0.42 E_{R}$ and $U_{12}=1.2 E_{R}$. These DNLSE parameters are derived from the intra- and interspecies scattering length of the species, the trap parameters and the width of the Gaussian basis, which is $0.3 a$. At zero temperature, the ground state of the TBEC has side-by-side geometry with $\Lambda=0$ [37]. Like in the case of ${ }^{87} \mathrm{Rb}^{-}{ }^{85} \mathrm{Rb}$, as the temperature of the TBEC is increased $\left(T<T_{c}\right)$, the system is transformed into the miscible phase. In addition, we have observed the bifurcation in the energy of the slosh mode, and the mode becomes degenerate with a discontinuity in the quasiparticle spectra at $T_{\mathrm{ch}}=0.62 T_{\mathrm{c}} \approx 140$ $\mathrm{nK}$. 


\section{B. ${ }^{87} \mathbf{R b}-{ }^{41} \mathbf{K}$ TBEC}

In the case of the ${ }^{87} \mathrm{Rb}-{ }^{41} \mathrm{~K}$ TBEC, the wavelength of the laser beams and the number of atoms are considered same as in the case of ${ }^{87} \mathrm{Rb}-{ }^{133} \mathrm{Cs}$ TBEC. The radial trapping frequencies are $\omega_{x}=\omega_{y}=\omega_{\perp}=2 \pi \times 100 \mathrm{~Hz}$ with the anisotropy parameter as 1.40 [88]. The tunneling matrix elements are $J_{1}=0.66 E_{R}$ and $J_{2}=2.84 E_{R}$ corresponding to $5 E_{R}$ lat- tice depth. The intraspecies and interspecies on-site interactions considered are $U_{11}=0.20 E_{R}, U_{22}=0.06 E_{R}$ and $U_{12}=0.60 E_{R}$. The set of parameters are chosen such that the density profile of the TBEC is immiscible and has side-byside geometry at zero temperature. Like in the previous cases, the geometry of the TBEC is transformed from side-by-side type to the rotationally symmetric overlapping profile and the slosh mode becomes degenerate at $T_{\mathrm{ch}}=0.53 T_{c} \approx 278 \mathrm{nK}$.
[1] D. Jaksch, C. Bruder, J. I. Cirac, C. W. Gardiner, and P. Zoller, Phys. Rev. Lett. 81, 3108 (1998).

[2] C. Orzel, A. K. Tuchman, M. L. Fenselau, M. Yasuda, and M. A. Kasevich, Science 291, 2386 (2001).

[3] M. Greiner, O. Mandel, T. Esslinger, T. W. Hänsch, and I. Bloch, Nature (London) 415, 39 (2002).

[4] I. Bloch, J. Dalibard, and S. Nascimbène, Nat. Phys. 8, 267 (2012).

[5] E. Demler and F. Zhou, Phys. Rev. Lett. 88, 163001 (2002).

[6] A. B. Kuklov and B. V. Svistunov, Phys. Rev. Lett. 90, 100401 (2003).

[7] A. Kuklov, N. Prokof'ev, and B. Svistunov, Phys. Rev. Lett. 92, 050402 (2004).

[8] A. Pal and D. A. Huse, Phys. Rev. B 82, 174411 (2010).

[9] S. Choi and L. Radzihovsky, Phys. Rev. A 84, 043612 (2011).

[10] H.-F. Lin, H.-D. Liu, H.-S. Tao, and W.-M. Liu, Sci. Rep. 5, 9810 (2015).

[11] O. Jürgensen, K. Sengstock, and D.-S. Lühmann, Sci. Rep. 5, 12912 (2015).

[12] S. Trotzky, P. Cheinet, S. Fölling, M. Feld, U. Schnorrberger, A. M. Rey, A. Polkovnikov, E. A. Demler, M. D. Lukin, and I. Bloch, Science 319, 295 (2008).

[13] J. Simon, W. S. Bakr, R. Ma, M. E. Tai, P. M. Preiss, and M. Greiner, Nature (London) 472, 307 (2011).

[14] I. Bloch, Nature (London) 453, 1016 (2008).

[15] M. Lewenstein, A. Sanpera, V. Ahufinger, B. Damski, A. Sen(De), and U. Sen, Adv. Phys. 56, 243 (2007).

[16] I. Bloch, J. Dalibard, and W. Zwerger, Rev. Mod. Phys. 80, 885 (2008).

[17] T. Mishra, R. V. Pai, and B. P. Das, Phys. Rev. A 76, 013604 (2007).

[18] F. Zhan and I. P. McCulloch, Phys. Rev. A 89, 057601 (2014).

[19] M. Greiner, I. Bloch, O. Mandel, T. W. Hänsch, and T. Esslinger, Phys. Rev. Lett. 87, 160405 (2001).

[20] T. Roscilde and J. I. Cirac, Phys. Rev. Lett. 98, 190402 (2007).

[21] P. Buonsante, S. M. Giampaolo, F. Illuminati, V. Penna, and A. Vezzani, Phys. Rev. Lett. 100, 240402 (2008).

[22] G. Ceccarelli, J. Nespolo, A. Pelissetto, and E. Vicari, Phys. Rev. A 92, 043613 (2015).

[23] G. Ceccarelli, J. Nespolo, A. Pelissetto, and E. Vicari, Phys. Rev. A 93, 033647 (2016).

[24] T.-L. Ho and V. B. Shenoy, Phys. Rev. Lett. 77, 3276 (1996).

[25] E. Timmermans, Phys. Rev. Lett. 81, 5718 (1998).

[26] B. D. Esry and C. H. Greene, Phys. Rev. A 59, 1457 (1999).

[27] L. Wen, W. M. Liu, Y. Cai, J. M. Zhang, and J. Hu, Phys. Rev. A 85, 043602 (2012).

[28] S. B. Papp, J. M. Pino, and C. E. Wieman, Phys. Rev. Lett. 101, 040402 (2008).

[29] S. Tojo, Y. Taguchi, Y. Masuyama, T. Hayashi, H. Saito, and T. Hirano, Phys. Rev. A 82, 033609 (2010).
[30] D. J. McCarron, H. W. Cho, D. L. Jenkin, M. P. Köppinger, and S. L. Cornish, Phys. Rev. A 84, 011603 (2011).

[31] G. V. Chester, Phys. Rev. 100, 446 (1955).

[32] M. D. Miller, Phys. Rev. B 18, 4730 (1978).

[33] J. Catani, L. De Sarlo, G. Barontini, F. Minardi, and M. Inguscio, Phys. Rev. A 77, 011603 (2008).

[34] B. Gadway, D. Pertot, R. Reimann, and D. Schneble, Phys. Rev. Lett. 105, 045303 (2010).

[35] P. Soltan-Panahi, J. Struck, P. Hauke, A. Bick, W. Plenkers, G. Meineke, C. Becker, P. Windpassinger, M. Lewenstein, and K. Sengstock, Nat. Phys. 7, 434 (2011).

[36] K. Suthar, A. Roy, and D. Angom, Phys. Rev. A 91, 043615 (2015).

[37] K. Suthar and D. Angom, Phys. Rev. A 93, 063608 (2016).

[38] F. Lingua, M. Guglielmino, V. Penna, and B. Capogrosso Sansone, Phys. Rev. A 92, 053610 (2015).

[39] P. N. Galteland, E. Babaev, and A. Sudbø, New J. Phys. 17, 103040 (2015).

[40] O. E. Alon, A. I. Streltsov, and L. S. Cederbaum, Phys. Rev. Lett. 97, 230403 (2006).

[41] O. E. Alon, A. I. Streltsov, and L. S. Cederbaum, Phys. Rev. A 76, 013611 (2007).

[42] O. E. Alon, A. I. Streltsov, and L. S. Cederbaum, Phys. Rev. A 76, 062501 (2007).

[43] O. E. Alon, A. I. Streltsov, and L. S. Cederbaum, Phys. Rev. A 77, 033613 (2008).

[44] L. S. Cederbaum, A. I. Streltsov, Y. B. Band, and O. E. Alon, Phys. Rev. Lett. 98, 110405 (2007).

[45] P. Öhberg, Phys. Rev. A 61, 013601 (1999).

[46] H. Shi, W.-M. Zheng, and S.-T. Chui, Phys. Rev. A 61, 063613 (2000).

[47] K. Nho and D. P. Landau, Phys. Rev. A 76, 053610 (2007).

[48] A. Roy and D. Angom, Phys. Rev. A 92, 011601 (2015).

[49] N. P. Proukakis, Phys. Rev. A 74, 053617 (2006).

[50] S. Dettmer, D. Hellweg, P. Ryytty, J. J. Arlt, W. Ertmer, K. Sengstock, D. S. Petrov, G. V. Shlyapnikov, H. Kreutzmann, L. Santos, and M. Lewenstein, Phys. Rev. Lett. 87, 160406 (2001).

[51] D. Hellweg, L. Cacciapuoti, M. Kottke, T. Schulte, K. Sengstock, W. Ertmer, and J. J. Arlt, Phys. Rev. Lett. 91, 010406 (2003).

[52] S. Richard, F. Gerbier, J. H. Thywissen, M. Hugbart, P. Bouyer, and A. Aspect, Phys. Rev. Lett. 91, 010405 (2003).

[53] J. Esteve, J.-B. Trebbia, T. Schumm, A. Aspect, C. I. Westbrook, and I. Bouchoule, Phys. Rev. Lett. 96, 130403 (2006).

[54] T. Plisson, B. Allard, M. Holzmann, G. Salomon, A. Aspect, P. Bouyer, and T. Bourdel, Phys. Rev. A 84, 061606 (2011).

[55] O. Penrose and L. Onsager, Phys. Rev. 104, 576 (1956).

[56] E. A. Burt, R. W. Ghrist, C. J. Myatt, M. J. Holland, E. A. Cornell, and C. E. Wieman, Phys. Rev. Lett. 79, 337 (1997). 
[57] B. Laburthe Tolra, K. M. O’Hara, J. H. Huckans, W. D. Phillips, S. L. Rolston, and J. V. Porto, Phys. Rev. Lett. 92, 190401 (2004).

[58] M. L. Chiofalo, M. Polini, and M. P. Tosi, Eur. Phys. J. D 11, 371 (2000).

[59] A. Smerzi and A. Trombettoni, Phys. Rev. A 68, 023613 (2003).

[60] M. P. A. Fisher, P. B. Weichman, G. Grinstein, and D. S. Fisher, Phys. Rev. B 40, 546 (1989).

[61] E. Lundh and J.-P. Martikainen, Phys. Rev. A 85, 023628 (2012).

[62] P. P. Hofer, C. Bruder, and V. M. Stojanović, Phys. Rev. A 86, 033627 (2012).

[63] A. Griffin, Phys. Rev. B 53, 9341 (1996).

[64] A. M. Rey, Ultracold bosonic atoms in optical lattices, Ph.D. thesis, University of Maryland, USA (2004).

[65] N. Elstner and H. Monien, Phys. Rev. B 59, 12184 (1999).

[66] S. Wessel, F. Alet, M. Troyer, and G. G. Batrouni, Phys. Rev. A 70, 053615 (2004).

[67] B. Capogrosso-Sansone, Ş. G. Söyler, N. Prokof'ev, and B. Svistunov, Phys. Rev. A 77, 015602 (2008).

[68] F. Gerbier, Phys. Rev. Lett. 99, 120405 (2007).

[69] E. Toth and P. B. Blakie, Phys. Rev. A 83, 021601 (2011).

[70] S. Klaiman, A. I. Streltsov, and O. E. Alon, Chem. Phys. 482, 362 (2017).

[71] W. Krauth, M. Caffarel, and J.-P. Bouchaud, Phys. Rev. B 45, 3137 (1992).

[72] V. A. Kashurnikov and B. V. Svistunov, Phys. Rev. B 53, 11776 (1996).
[73] M. Capello, F. Becca, M. Fabrizio, and S. Sorella, Phys. Rev. Lett. 99, 056402 (2007).

[74] S. Ramanan, T. Mishra, M. S. Luthra, R. V. Pai, and B. P. Das, Phys. Rev. A 79, 013625 (2009).

[75] V. A. Kashurnikov, N. V. Prokof'ev, and B. V. Svistunov, Phys. Rev. A 66, 031601 (2002).

[76] A. Isacsson, M.-C. Cha, K. Sengupta, and S. M. Girvin, Phys. Rev. B 72, 184507 (2005).

[77] R. A. Barankov, C. Lannert, and S. Vishveshwara, Phys. Rev. A 75, 063622 (2007).

[78] K. Mitra, C. J. Williams, and C. A. R. Sá de Melo, Phys. Rev. A 77, 033607 (2008).

[79] R. J. Glauber, Phys. Rev. 131, 2766 (1963).

[80] M. Naraschewski and R. J. Glauber, Phys. Rev. A 59, 4595 (1999).

[81] A. Bezett, E. Toth, and P. B. Blakie, Phys. Rev. A 77, 023602 (2008).

[82] A. Bezett and E. Lundh, J. Phys. B 45, 205301 (2012).

[83] E. Anderson, Z. Bai, C. Bischof, S. Blackford, J. Demmel, J. Dongarra, J. D. Croz, A. Greenbaum, S. Hammarling, A. McKenney, and D. Sorensen, LAPACK Users' Guide, 3rd ed. (Society for Industrial and Applied Mathematics, Philadelphia, PA, 1999).

[84] D. Baillie and P. B. Blakie, Phys. Rev. A 80, 031603 (2009).

[85] C. Ticknor, Phys. Rev. A 88, 013623 (2013).

[86] C. Ticknor, Phys. Rev. A 89, 053601 (2014).

[87] C. Gies and D. A. W. Hutchinson, Phys. Rev. A 70, 043606 (2004).

[88] G. Thalhammer, G. Barontini, L. De Sarlo, J. Catani, F. Minardi, and M. Inguscio, Phys. Rev. Lett. 100, 210402 (2008). 